\title{
COTOVELO FLUTUANTE EM CRIANÇAS
}

\author{
FLOATING ELBOW IN CHILDREN
}

Jamil Faissal Soni', Weverley Rubele Valenza ${ }^{2}$, Gisele Cristine Schelle ${ }^{3}$, Dalton Berri ${ }^{3}$, Anna Carolina Pavelec ${ }^{4}$

\section{RESUMO}

Objetivo: Analisar, retrospectivamente, o manejo do cotovelo flutuante em crianças, atendidas no Hospital do Trabalhador - UFPR, com enfoque na ocorrência de lesões associadas, tratamento realizado e resultados obtidos. Métodos: Entre abril de 2002 e abril de 2007, foram atendidos 15 pacientes com cotovelo flutuante, idade entre três e 14 anos, 12 pacientes do sexo masculino e três do feminino, oito fraturas no membro superior direito e sete no esquerdo, com seguimento mínimo de três anos e oito meses. Avaliamos, ainda, o mecanismo do trauma, lesões associadas, exposição da fratura, classificação, tratamento e os resultados. Resultados: Sete pacientes sofreram queda da mesma altura; três, queda de nível; um, do cavalo; um, de motocicleta; uma criança atropelada; e duas prenderam o braço na centrífuga. No úmero tivemos quatro fraturas diafisárias e 11 fraturas supracondilianas. No antebraço, 13 fraturas do $1 / 3$ distal e duas diafisárias. Tivemos três fraturas expostas, dois pacientes com lesão nervosa, um com síndrome compartimental e nenhum apresentou lesão vascular. Um paciente foi tratado com tala gessada, sendo que os outros 14 pacientes tiveram suas fraturas fixadas tanto no úmero quanto no antebraço. Tivemos um paciente que evoluiu com varo de cinco graus e não tivemos alterações funcionais na nossa série. Conclusão: O cotovelo flutuante é uma lesão infrequente, com potencial risco de complicações, entre elas: lesões nervosas, exposição óssea e síndrome compartimental. Recomendamos para seu tratamento, redução e fixação de ambas as fraturas, o que permite melhor cuidado das partes moles, bem como avaliação da perfusão do membro, com bons resultados funcionais.

\section{ABSTRACT}

Objective: To analyze the management of floating elbow injuries in children at hospital do Trabalhador- UFPR, focusing on associated lesions, treatment and results. Methods: Between April 2002 to April 2007, 15 patients with floating elbow were treated at the same hospital service. Age ranged from 3 to 14 years old and 12 were boys. Eight fractures were at right and 7 at left side. The minimal follow up time was 44 months. We also evaluated the trauma mechanism, associated lesions, fracture exposition, classification, treatment and results. Results: In seven patients the trauma was the result of a same level fall and 3 of a fall from a height, one had a horseback fall, one from a motorcycle, one was hit by a car and 2 had the limb stuck in a washing machine. At the humerus we have 4 diaphyseal and 11 supracondylar fractures. At forearm we have 13 distal and two diaphyseal fractures. We have 3 open fractures, 2 patients with neurologic injuries, 1 with compartment syndrome, and none patient with vascular injury. One patient was treated with splint cast the other 14 patients underwent fracture fixation for humerus and forearm. One patient had a 5 degree varus deviation and there was no functional compromise. Conclusions: The floating elbow is rare, but it has a high potential for complications like nerve lesions, bone exposition and compartment syndrome. We recommend reduction and fixation of both fractures allowing better care to the soft tissue around, it also provides better evaluation of limb vascular perfusion and good functional results.

Keywords - Fracture Fixation; Child; Elbow Joint

\section{INTRODUÇÃO}

A ocorrência simultânea entre fratura do úmero com fratura ipsilateral do antebraço, na população pediátrica, foi primeiramente denominada de cotovelo flutuante por Stanitski e Micheli ${ }^{(1)}$. Esta lesão é incomum, com sua incidência variando entre dois a $13 \%$, sendo a fratura

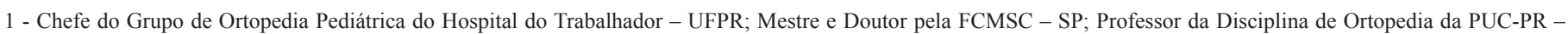
Curitiba, PR, Brasil.

2 - Médico Assistente do Grupo de Ortopedia Pediátrica do Hospital do Trabalhador - UFPR - Curitiba, PR, Brasil.

3 - Médica Assistente do Grupo de Ortopedia Pediátrica do Hospital do Trabalhador - UFPR - Curitiba, PR, Brasil.

4 - Médica Assistente do Grupo de Ortopedia Pediátrica do Hospital Universitário Cajuru - PUC-PR - Curitiba, PR, Brasil.

Trabalho realizado no Serviço de Ortopedia e Traumatologia do Hospital do Trabalhador - UFPR, Prof. Dr. Marcelo Abagge - Curitiba, PR.

Correspondência: Avenida Silva Jardim, 1.502, ap. 91, - 80250-200 - Curitiba, PR. E-mail: jamilfsoni@hotmail.com.

Trabalho recebido para publicação: 24/08/2011, aceito para publicação: 31/08/2011.

Os autores declaram inexistência de conflito de interesses na realização deste trabalho / The authors declare that there was no conflict of interest in conducting this work 
supracondiliana do úmero frequentemente encontrada como a lesão proximal ao cotovelo ${ }^{(1)}$.

O cotovelo flutuante é considerado como um indicador de trauma de grande energia, podendo ocorrer concomitantemente a fratura exposta, lesões nervosas, lesões vasculares e síndrome compartimental ${ }^{(2,3)}$.

No passado, muitos autores postulavam a abordagem incruenta para estas lesões; porém, recentemente, a indicação cirúrgica tem prevalecido ${ }^{(1-5)}$.

O objetivo deste trabalho é analisar o manejo do cotovelo flutuante na população pediátrica, em pacientes atendidos no Hospital do Trabalhador - UFPR, com enfoque nas ocorrências de lesões associadas, tratamento realizado e resultados obtidos.

\section{CASUÍSTICA E MÉTODO}

No período compreendido entre abril de 2002 e abril de 2007 foram atendidos, no Serviço de Ortopedia e Traumatologia do Hospital do Trabalhador - UFPR, 15 pacientes esqueleticamente imaturos com cotovelo flutuante, na faixa etária de três a 14 anos (média = oito anos), sendo sete fraturas comprometendo o membro superior esquerdo e oito, o direito; 12 pacientes eram do sexo masculino e três, do feminino. O seguimento mínimo foi de três anos e oito meses e o máximo, de sete anos e oito meses (Tabela 1).

Na Tabela 2 descrevemos o mecanismo de lesão, presença de exposição classificadas segundo Gustilo e Anderson $^{(6)}$, para a fratura do antebraço e a do úmero foram classificadas segundo pelo método $\mathrm{AO} / \mathrm{ASIF}$, para as

Tabela 1 - População estudada.

\begin{tabular}{c|c|c|c|c}
\hline Casos & Sexo & Idade & Lado & Data \\
\hline 1 & $\mathrm{M}$ & 9 & $\mathrm{E}$ & $25 / 02 / 2004$ \\
\hline 2 & $\mathrm{~F}$ & 9 & $\mathrm{E}$ & $16 / 11 / 2004$ \\
\hline 3 & $\mathrm{M}$ & 3 & $\mathrm{D}$ & $17 / 02 / 2004$ \\
\hline 4 & $\mathrm{M}$ & 6 & $\mathrm{E}$ & $01 / 08 / 2004$ \\
\hline 5 & $\mathrm{M}$ & 8 & $\mathrm{D}$ & $02 / 11 / 2003$ \\
\hline 6 & $\mathrm{M}$ & 14 & $\mathrm{E}$ & $02 / 04 / 2003$ \\
\hline 7 & $\mathrm{M}$ & 4 & $\mathrm{D}$ & $12 / 04 / 2002$ \\
\hline 8 & $\mathrm{M}$ & 9 & $\mathrm{D}$ & $26 / 08 / 2004$ \\
\hline 9 & $\mathrm{~F}$ & 6 & $\mathrm{E}$ & $08 / 07 / 2005$ \\
\hline 10 & $\mathrm{M}$ & 8 & $\mathrm{E}$ & $12 / 01 / 2004$ \\
\hline 11 & $\mathrm{M}$ & 10 & $\mathrm{D}$ & $10 / 06 / 2004$ \\
\hline 12 & $\mathrm{~F}$ & 10 & $\mathrm{E}$ & $10 / 02 / 2004$ \\
\hline 13 & $\mathrm{M}$ & 7 & $\mathrm{D}$ & $02 / 03 / 2005$ \\
\hline 14 & $\mathrm{M}$ & 12 & $\mathrm{D}$ & $06 / 10 / 2006$ \\
\hline 15 & $\mathrm{M}$ & 9 & $\mathrm{D}$ & $04 / 04 / 2007$ \\
\hline
\end{tabular}

fraturas pediátricas ${ }^{(7)}$ e na eventualidade de comprometer a região supracondiliana do úmero foram classificadas pela classificação de Gartland $^{(8)}$. O tratamento instituído, tanto para a fratura do úmero quanto a do antebraço, foi descrito, para cada caso, segundo o método aplicado. A presença de lesões associadas, tais como lesões vasculares, neurológicas e síndrome compartimental, bem como os resultados finais referindo-se à consolidação, presença de limitação funcional e deformidades angulares residuais estão listados na Tabela 3.

\section{RESULTADOS}

Tivemos sete pacientes vítimas de queda da própria altura, três sofreram queda de nível, um do cavalo e outro sofreu queda de motocicleta, uma criança foi atropelada e dois apresentaram a lesão por prender o membro superior em máquina de centrífuga.

No úmero encontramos quatro fraturas diafisárias: duas 12-D5.1 e duas 12-D4.1 pela classificação AO, 10 fraturas supracondilianas Gartland tipo III e uma, Gartland II (Figura 1). As fraturas do antebraço eram 13 do 1/3 distal do antebraço, uma lesão de Galleazi (22$\mathrm{D} / 7.1)$, uma subperiosteal (23r-D/2.1), quatro descolamentos epifisários (23-E/2.1), sete fraturas completas com desvio (23-M/2.1) e duas fraturas diafisárias do antebraço (22-D/1.1 e 22-D/5.1).

Tivemos três fraturas consideradas expostas e todas classificadas como grau I, segundo Gustilo e Anderson, uma fratura supracondiliana do úmero (paciente 11), uma diafisária do úmero (paciente 12) e uma metafisária do antebraço (paciente 13).

O tratamento instituído para a fratura do úmero foi incruento, com imobilização gessada em apenas um paciente, caso número 7 . Os 14 restantes foram tratados por redução e fixação tanto da fratura do antebraço como do úmero, sendo que em dois (pacientes 9 e 14) utilizamos a técnica de fixação intramedulares flexíveis com fios de Kirschner, e um deles (paciente 12) recebeu placa de compressão na fratura do úmero e fio na fratura distal (Galleazi) (Figura 2).

Para as fraturas do antebraço, uma foi tratada conservadoramente, fratura subperiosteal, dois pacientes receberam fixação intramedular, e o restante fixado localmente.

Foram observados dois pacientes com comprometimento neurológico inicial, sendo um do nervo interósseo posterior (caso 1) e outro com acometimento do mediano (caso 10), ambas recuperaram espontaneamente durante os primeiros meses. Um paciente evoluiu com 
Tabela 2 - Mecanismo de lesão, classificação e tratamento.

\begin{tabular}{|c|c|c|c|c|c|c|}
\hline Casos & Mecanismo & Classif. punho & Classif. úmero & Trat. úmero & Trat. antebraço & Exposta \\
\hline 1 & Queda propria altura & 23-M/3.1 & Gartland III & 3 fios $K$. laterais & 2 fios K. cruzados & $\mathrm{N}$ \\
\hline 2 & Queda de nivel (1m.) & 23-M/3.1 & Gartland III & 3 fios $K$. laterais & 1 fio $K$. & $\mathrm{N}$ \\
\hline 3 & Queda mesma altura & 23-M/3.1 & Gartland III & 3 fios $K$. laterais & 1 fio $K$. & $\mathrm{N}$ \\
\hline 4 & Queda mesma altura & $23-M / 3.1$ & Gartland III & 2 fios $K$. laterais & 1 fio $K$. & $\mathrm{N}$ \\
\hline 5 & Queda da escada & $23-E / 2.1$ & Gartland III & 3 fios K. cruzados & 1 fio $K$. & $\mathrm{N}$ \\
\hline 6 & Atropelamento & $23-E / 2.1$ & Gartland III & 2 fios $K$. laterais & 1 fio $K$. & $\mathrm{N}$ \\
\hline 7 & Centrífuga & $23 r-M / 2.1$ & $12-D / 5.1$ & Gesso BP & Gesso BP & $\mathrm{N}$ \\
\hline 8 & Queda do cavalo & 23-M/3.1 & Gartland II & 2 fios $K$. laterais & 1 fio $K$. & $\mathrm{N}$ \\
\hline 9 & Prendeu centrífuga & $22-D / 5.1$ & $12-\mathrm{D} / 5.1$ & 2 fios I.M. & Fios I.M. & $\mathrm{N}$ \\
\hline 10 & Queda própria altura & $23-E / 2.1$ & Gartland III & 3 fios $K$. laterais & 1 fio $K$. & $\mathrm{N}$ \\
\hline 11 & Queda da escada & $23-E / 2.1$ & Gartland III & Fios K. cruzados & 1 fio $K$. & S. grau I \\
\hline 12 & Queda de moto & $22-\mathrm{D} / 7.1$ & $12-\mathrm{D} / 4.1$ & Placa e parafuso & 1 fio $K$. & S. grau I \\
\hline 13 & Queda mesma altura & 23-M/3.1 & Gartland III & Fios K. cruzados & Fio K. radio e ulna & S. grau I \\
\hline 14 & Queda mesma altura & $22-D / 1.1$ & $12-D / 4.1$ & 2 fios I.M. & Fios I.M. & $\mathrm{N}$ \\
\hline 15 & Queda mesma altura & 23-M/3.1 & Gartland III & 2 fios $K$. laterais & 1 fio $K$. & $\mathrm{N}$ \\
\hline
\end{tabular}

Tabela 3 - Resultados e complicações.

\begin{tabular}{c|c|c|c|c|c}
\hline Casos & I vasc. & I. nervosa & $\begin{array}{c}\text { Sínd. } \\
\text { compart. }\end{array}$ & $\begin{array}{c}\text { Resultado } \\
\text { funcional }\end{array}$ & $\begin{array}{c}\text { Deformidade } \\
\text { angular } \\
\text { residual }\end{array}$ \\
\hline 1 & $\mathrm{~N}$ & $\begin{array}{c}\text { Interósseo } \\
\text { posterior }\end{array}$ & $\mathrm{N}$ & Normal & Ausente \\
\hline 2 & $\mathrm{~N}$ & $\mathrm{~N}$ & $\mathrm{~N}$ & Normal & Ausente \\
\hline 3 & $\mathrm{~N}$ & $\mathrm{~N}$ & $\mathrm{~N}$ & Normal & Ausente \\
\hline 4 & $\mathrm{~N}$ & $\mathrm{~N}$ & $\mathrm{~N}$ & Normal & Ausente \\
\hline 5 & $\mathrm{~N}$ & $\mathrm{~N}$ & $\mathrm{~N}$ & Normal & Ausente \\
\hline 6 & $\mathrm{~N}$ & $\mathrm{~N}$ & $\mathrm{~N}$ & Normal & Ausente \\
\hline 7 & $\mathrm{~N}$ & $\mathrm{~N}$ & $\mathrm{~N}$ & Normal & Ausente \\
\hline 8 & $\mathrm{~N}$ & $\mathrm{~N}$ & $\mathrm{~N}$ & Normal & Ausente \\
\hline 9 & $\mathrm{~N}$ & $\mathrm{~N}$ & $\mathrm{~N}$ & Normal & Ausente \\
\hline 10 & $\mathrm{~N}$ & $\mathrm{Mediano}$ & $\mathrm{N}$ & Normal & Ausente \\
\hline 11 & $\mathrm{~N}$ & $\mathrm{~N}$ & $\mathrm{~N}$ & Normal & Ausente \\
\hline 12 & $\mathrm{~N}$ & $\mathrm{~N}$ & $\mathrm{~S}$ & Normal & Ausente \\
\hline 13 & $\mathrm{~N}$ & $\mathrm{~N}$ & $\mathrm{~N}$ & Normal & Varo 5 graus \\
\hline 14 & $\mathrm{~N}$ & $\mathrm{~N}$ & $\mathrm{~N}$ & Normal & Ausente \\
\hline 15 & $\mathrm{~N}$ & $\mathrm{~N}$ & $\mathrm{~N}$ & Normal & Ausente \\
\hline
\end{tabular}

síndrome compartimental (caso 12), realizada fasciotomia e fixação com placa no úmero que evoluiu sem sequelas.

Não observamos lesão vascular inicial em nossa amostra.

Não houve alterações quanto à limitação do movimento tanto quanto o cotovelo ou punho, quando comparado ao lado contralateral. Um paciente (caso 13) com fratura supracondiliana grau III e metafisária completa do rádio evoluiu com cúbito varo de cinco graus, que permanece em observação, sem queixas estéticas ou funcionais.

\section{DISCUSSÃO}

Muitos autores recomendavam em seus relatos o tratamento conservador para as duas fraturas e o tratamento pela tração olecraniana ou fixação com fios de Kirschner da fratura supracondiliana do úmero ${ }^{(1,4,9,10)}$. Com uma melhor compreensão da personalidade da fratura, da possibilidade de síndrome compartimental, lesão neurológica e vascular, de perda de redução e de consolidação viciosa os autores tendem ao tratamento cirúrgico com fixação de ambas as fraturas ${ }^{(3,5)}$.

Torna-se, portanto, importante diferenciar as fraturas de baixa energia das de maior energia, sendo que os traumas menores com fraturas sem desvios podem ser tratadas com gessos circulares, pois dificilmente evoluem para síndrome compartimental, ou podem ser imobilizadas com tala gessada sem grandes riscos de desvios posteriores e consolidação viciosa.

Nas fraturas completas, com maior energia do trauma, aumenta o risco de síndrome compartimental, sendo que, em nossa série, tivemos um paciente que necessitou fasciotomia. O trauma proporciona uma maior lesão de partes moles e tivemos, ainda, dois pacientes com neuropraxia e três fraturas expostas, o que nos leva a crer que o aparelho gessado circular não é uma boa opção para estes pacientes, pois inviabiliza um bom exame neurovascular do membro fraturado.

Além disso, o edema de partes moles pode fazer com que o gesso provoque uma síndrome compartimental, como relatado na série de Ring et $a l^{(3)}$, que tiveram duas 


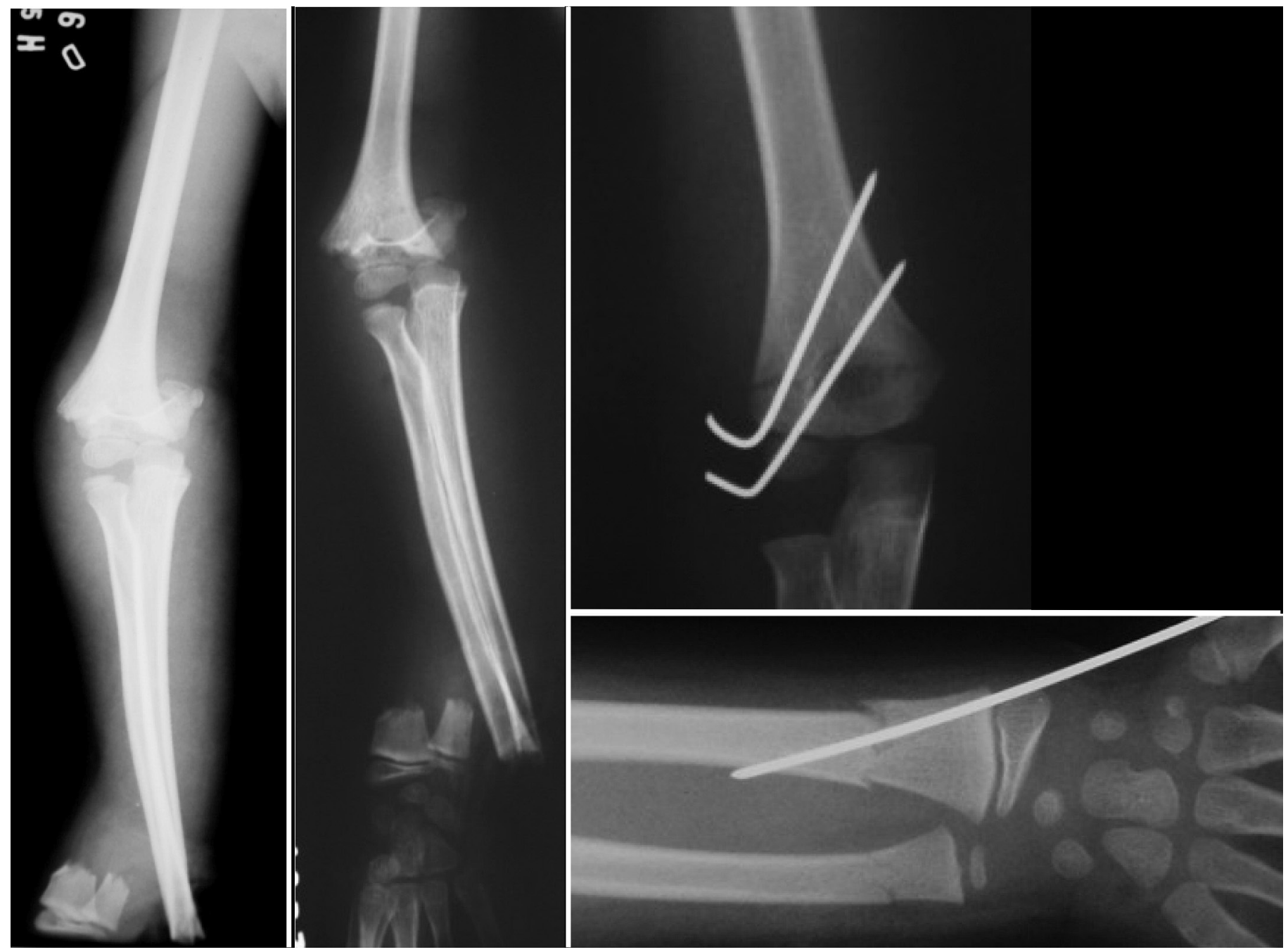

Figura 1 - Paciente 4, sexo masculino, seis anos, fratura supracondiliana do úmero e do antebraço distal esquerdo, queda do mesmo nível.

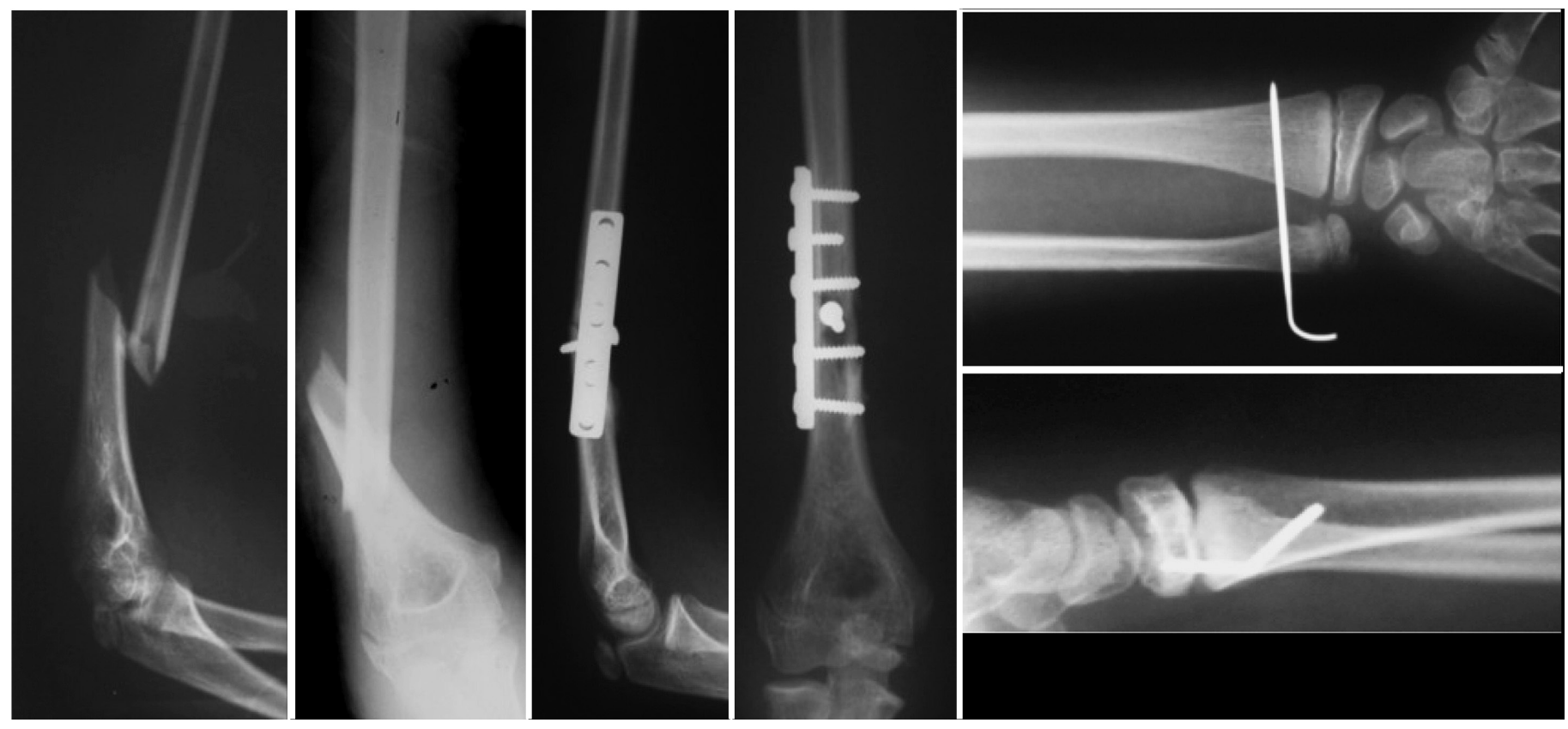

Figura 2 - Paciente 12, sexo feminino, 10 anos, fratura exposta grau I do úmero esquerdo e fratura Galleazi do antebraço esquerdo. Fixação com placa e parafuso no úmero e com fio de Kirschner no antebraço. 
síndromes compartimentais por compressão extrínseca, que resolveram com a remoção do gesso.

Após a regressão do edema inicial, as fraturas imobilizadas com gesso circular podem sofrer desvios posteriores, sendo necessária uma nova redução ou fixação para evitar a consolidação viciosa.

Acreditamos que há espaço para o tratamento conservador nas fraturas de baixa energia, sem desvio e que para as fraturas desviadas a fixação de ambas as fraturas do cotovelo flutuante proporciona controle adequado dos fragmentos e menor risco de complicações.

Em nossa série não foi observada alteração de mobilidade comparando-se com o lado contralateral; tivemos um paciente com varo de cinco graus, resultado de uma redução inicial inadequada. Um paciente que se apresentou com síndrome compartimental e três apresentaram fraturas expostas e a estabilização destas permitiu um melhor cuidado das partes moles. Não foi encontrada presença de síndrome compartimental após a estabili- zação das fraturas; acreditamos que a fixação permite um melhor acompanhamento pós-operatório.

Quanto ao local, a fratura supracondiliana é a mais frequente no segmento umeral. Tivemos 11 pacientes com fraturas supracondilianas e quatro com fraturas diafisárias do úmero. No antebraço foram três diafisárias, 12 acometendo o terço distal sendo extremamente raro o comprometimento proximal do antebraço, de acordo com as observações de Ríos e Lúcio ${ }^{(11)}$.

\section{CONCLUSÕES}

O cotovelo flutuante é uma lesão infrequente, com potencial risco de complicações, entre elas as lesões nervosas e a síndrome compartimental. Recomendamos, para seu tratamento, redução e fixação de ambas as fraturas, o que permite melhor cuidado das partes moles, bem como avaliação da perfusão do membro, com bons resultados funcionais.

\section{REFERÊNCIAS}

1. Stanitski CL, Micheli LJ. Simultaneous ipsilateral fractures of the arm and forearm in children. Clin Orthop Relat Res. 1980;(153):218-22.

2. Harrington P, Sharif I, Fogarty EE, Dowling FE, Moore DP. Management of the floating elbow injury in children. Simultaneous ipsilateral fractures of the elbow and forearm. Arch Orthop Trauma Surg. 2000;120(3-4):205-8.

3. Ring D, Waters PM, Hotchkiss RN, Kasser JR. Pediatric floating elbow. J Pediatr Orthop. 2001;21(4):456-9.

4. Reed FE Jr, Apple DF Jr. Ipsilateral fractures of the elbow and forearm. SouthMed J. 1976;69(2):149-51.

5. Templeton PA, Graham HK. The 'floating elbow' in children. Simultaneous supracondylar fractures of the humerus and of the forearm in the same upper limb.J Bone Joint Surg Br. 1995;77(5):791-6.

6. Gustilo RB, Anderson JT. Prevention of infection in the treatment of one thou- sand and twenty-five open fractures of long bones: retrospective and prospective analyses. J Bone Joint Surg Am. 1976;58(4):453-8.

7. Slongo T, Audigé L, Clavert JM, Lutz N, Frick S, Hunter J. The AO comprehensive classification of pediatric long-bone fractures: a web-based multicenter agreement study. J Pediatr Orthop. 2007;27(2):171-80.

8. Gartland JJ. Management of supracondylar fractures of the humerus in children. Surg Gynecol Obstet. 1959;109(2):145-54.

9. Papavasiliou $V$, Nenopoulos $S$. Ipsilateral injuries of the elbow and forearm in children. J Pediatr Orthop. 1986;6(1):58-60.

10. Biyani A, Gupta SP, Sharma JC. Ipsilateral supracondylar fracture of humerus and forearm bones in children. Injury. 1989;20(4):203-7.

11. Ríos EC, Lucio LN. [A prognostic classification of the "floating elbow" in children]. Acta Ortop Mex. 2007;21(6):300-3. 